FOLIA

Amazónica

Revista del Instituto de Investigaciones

de la Amazonía Peruana

\title{
HUELLA DE CARBONO DE LA VENTA DEL FRUTO Y LA PRODUCCIÓN DE BEBIDAS Y HELADOS DE AGUAJE (Mauritia flexuosa L.f.) EN EL DEPARTAMENTO DE UCAYALI, PERÚ
}

\author{
Diego GARCIA SORIA ${ }^{1 *}$, Jorge DELGADO SAENZ², Jorge REVILLA CHÁVEZ", \\ Jhon DEL AGUILA PASQUEL ${ }^{1}$, Eurídice HONORIO CORONADO ${ }^{1}$, \\ Dennis DEL CASTILLO ${ }^{1}$, Wilson GUERRA AREVALO ${ }^{1}$, Enrique GARCIA PEIXOTO ${ }^{3}$ \\ 1 Instituto de Investigaciones de la Amazonía Peruana. \\ 2 Universidad Nacional de Ucayali, Facultad de Ciencias Forestales y Ambientales. Pucallpa, Perú. \\ ${ }^{3}$ Colegio de Ingenieros del Perú, CIP. Capítulo de Ingenieros Agrónomos. Pucallpa, Perú. \\ * Correo electrónico: dgarcia@iiap.gob.pe
}

\section{RESUMEN}

Se estimó la huella de carbono de la venta del fruto, la producción de bebidas y helados de aguaje. El estudio se realizó en la ciudad de Pucallpa, distrito de Callería, donde se venden los productos del aguaje y en el caserío Túpac Amaru, distrito de Manantay donde se extraen los frutos del aguaje, ambos lugares ubicados en la provincia de Coronel Portillo, departamento de Ucayali. Se estudió la huella de carbono de todas las fases de la comercialización de los frutos, así como de la producción de bebidas y helados de aguaje, para lo cual se colectó información sobre las emisiones de $\mathrm{CO}_{2}$, a través de encuestas realizadas a 46 productores. Se estimó que la huella de carbono de la venta del fruto de aguaje fue de $0,07031 \mathrm{KgCO}_{2}$ eq por cada fruto obtenido talando la palmera y puesto en los centros de venta minoristas de la ciudad de Pucallpa. Las emisiones fueron menores cuando el fruto fue aprovechado sin talar la palmera, reduciendo la huella de carbono en un 83,53 \%, es decir, emitiendo solo 0,001158 $\mathrm{KgCO}_{2}$ eq por fruto de aguaje. Las huellas de carbono de un vaso de bebida de aguaje de $250 \mathrm{ml}$ y del helado de aguaje de $75 \mathrm{ml}$ fueron mayores, obteniéndose valores de 0,89317 y $0,0887 \mathrm{KgCO}_{2}$ eq, respectivamente. Por lo tanto, existe la necesidad de impulsar el aprovechamiento sostenible del aguaje utilizando tecnología 
que permitan subir las palmeras sin tener que talarla. Dicha tecnología deberá ser aceptada por los pobladores que realizan la extracción y favorecida por los consumidores finales.

PALABRAS CLAVE: Amazonía, ciclo de vida, emisiones de $\mathrm{CO}_{2}$, extracción de aguaje.

\title{
CARBON FOOTPRINT FROM THE SALE OF THE FRUIT AND THE PRODUCTION OF AGUAJE (Mauritia flexuosa L. f.) DRINKS AND ICE CREAMS IN THE DEPARTMENT OF UCAYALI, PERU
}

\begin{abstract}
The carbon footprint of the sale of the fruit and the production beverages and ice creams of aguaje was estimated. The study was carried out in the city of Pucallpa, Callería district, where the products are sold, and in the Túpac Amaru hamlet, Manantay district where the fruits are extracted, which are both located in the province of Coronel Portillo, department of Ucayali. The carbon footprint of all the phases of the commercialization of the fruits, and the production of drinks and aguaje ice creams were studied, for which information on their $\mathrm{CO}_{2}$ emissions was collected through surveys carried out to 46 producers. It was estimated that the carbon footprint of the sale of the aguaje fruit was 0.07031 $\mathrm{KgCO}_{2}$ eq for each fruit obtained by felling the palm tree and placed in the retail centers of the city of Pucallpa. Emissions were lower when the fruit was harvested without cutting down the palm tree, reducing the carbon footprint by $83.53 \%$, that is, emitting only $0.001158 \mathrm{KgCO}_{2}$ eq per aguaje fruit. The carbon footprints of a $250 \mathrm{ml}$ glass of aguaje drink and the $75 \mathrm{ml}$ aguaje ice cream were higher, obtaining values of 0.89317 and $0.0887 \mathrm{KgCO}_{2}$ eq, respectively. Therefore, there is a need to promote the use of the sustainable use of aguaje using technology that allows the palm trees to be ascended without having to cut them down. This technology must be accepted by the inhabitants who carry out the extraction and favored by the consumers.
\end{abstract}

KEYWORDS: Amazon, life cycle, $\mathrm{CO}_{2}$ emissions, aguaje extraction. 


\section{INTRODUCCIÓN}

Las emisiones de gases de efecto invernadero (GEI) de diversas actividades antrópicas siguen en aumento en el mundo (OMM, 2019), poniendo en debate las estrategias de mitigación del cambio climático para evitar las emisiones (Licona \& Ramírez, 2014). La venta de productos en los mercados nacionales e internacionales tiene un gran impacto en las emisiones del país, por lo que es necesario determinar con mayor precisión qué actividades presentan mayores emisiones en el proceso productivo y, al mismo tiempo, proponer estrategias de mitigación. En este contexto, empresas en todo el mundo estiman sus emisiones de GEI a través de la "huella de carbono" de sus productos, proponiendo medidas para reducir estas emisiones (Lash \& Wellington, 2007). En este sentido, contar con la huella de carbono de un producto puede influir en la preferencia de los consumidores a través de sus decisiones de compra, además, una amplia estimación de la huella de carbono, incluyendo los efectos de la cadena de suministro, es apropiada para una estimación más precisa. Igualmente puede motivar un clima corporativo más efectivo que motive el cambio en sus políticas de tratamiento de emisiones (Matthews et al., 2008). Por ello, la huella de carbono es la herramienta utilizada para determinar estrategias de mitigación de las emisiones de una empresa. Sin embargo, la decisión sobre la importancia de la huella de carbono de un producto, recae en el consumidor final.

En los bosques amazónicos del territorio peruano existen áreas pantanosas dominadas por la palmera de aguaje (Mauritia flexuosa L.f.) que son conocidas como aguajales. Estos aguajales alcanzan una superficie de 6'063.551,00 de hectáreas, siendo zonas que permanecen inundadas la mayor parte del tiempo (MINAM, 2010). En ese sentido, los aguajales juegan un rol importante desde el punto de vista económico, social y ambiental en la Amazonía peruana, estando estrechamente vinculados a la vida y cultura de los pueblos amazónicos (Freitas et al., 2006). Por otro lado, los suelos de los ecosistemas de aguajal de zonas bajas de Ucayali, constituyen importantes sumideros de carbono y contienen un total de 304,6 toneladas de carbono por hectárea (tC ha-1) (García et al., 2012).

El principal producto aprovechable del aguajal es el fruto del aguaje, que tiene alto contenido de vitamina A; alrededor de 30 miligramos (mg) por 100 gramos (g) de pulpa (Santos, 2005). Los frutos del aguaje son consumidos de forma directa como fruta fresca, como bebida (aguajina), pulpa, heladosy chupetes (Rojas, 2001). La alta demanda de frutos del aguaje en las ciudades amazónicas, afecta de manera directa a los aguajales, debido a que la cosecha de los frutos se realiza talando las palmeras femeninas que producen los frutos. Se calcula que la demanda de aguaje de la ciudad de Iquitos es de aproximadamente 21,9 toneladas (t) por día, lo que implica que diariamente se cortan unas 12.000 palmeras femeninas del medio natural para abastecer el mercado (García, 2001; Del Castillo et al., 2006; Delgado, 2007).

Ante esta alarmante situación es necesario cuantificar el impacto de las actividades dentro del ciclo productivo de los frutos y derivados del aguaje, sobre todo el impacto que genera la tala de los individuos femeninos para la extracción del fruto. El estudio tuvo como objetivo estimar la huella de carbono causada por la venta del fruto y la producción de refresco y helado de aguaje en el departamento de Ucayali, Perú. 


\section{MATERIALES Y MÉTODOS}

\section{ÁREA DE ESTUDIO}

El presente estudio se realizó en la ciudad de Pucallpa, distrito de Callería, y en el caserío Túpac Amaru, distrito de Manantay, ubicados en las coordenadas $8^{\circ} 24^{\prime} 21^{\prime \prime S} 74^{\circ} 34^{\prime} 42^{\prime \prime} 0$ y $8^{\circ} 28^{\prime} 31^{\prime \prime S}$ $74^{\circ} 36^{\prime} 39^{\prime \prime} 0$, respectivamente, en la provincia de Coronel Portillo, departamento de Ucayali, Perú (Figura 1). En la ciudad de Pucallpa se colectó la información referida a las actividades del transporte, preparación, almacenaje y comercialización del aguaje en fruto, bebida y helado. En el caserío Túpac Amaru se colectó información referida a las actividades de aprovechamiento, almacenaje y transporte de los frutos hacia Pucallpa para su comercialización.

\section{CICLO DEVIDA DE LACADENADE SUMINISTRO DEL AGUAJE}

Para el presente estudio se usó el modelo de evaluación del ciclo de vida (LCA, siglas en ingles), utilizando los métodos de entrada-salida que rastrean todas las actividades a través de la cadena de suministro para una industria específica. En este estudio, este ciclo se inicia desde la extracción del fruto en los aguajales, pasando por el trasporte y procesamiento de los diferentes productos, hasta su comercialización en los mercados locales. Los modelos de entradasalida fueron propuestos por primera vez por Leontief (1986) para ayudar en la planificación de la fabricación de cualquier producto.

\section{ENCUESTAS ESTRUCTURADAS}

La colecta de información para calcular la huella de carbono de la producción del fruto, bebida y helado a base de aguaje en el departamento de Ucayali, Perú, se llevó a cabo a través de encuestas diseñadas con base en visitas previas a los productores, transportistas, comercializadores y establecimientos de venta, complementadas por los trabajos realizados por DEFRA (2008). Las encuestas fueron diseñadas con preguntas que facilitaran el recuerdo de las cantidades. Por ello, se optó por indagar respuestas con cuantías diarias.

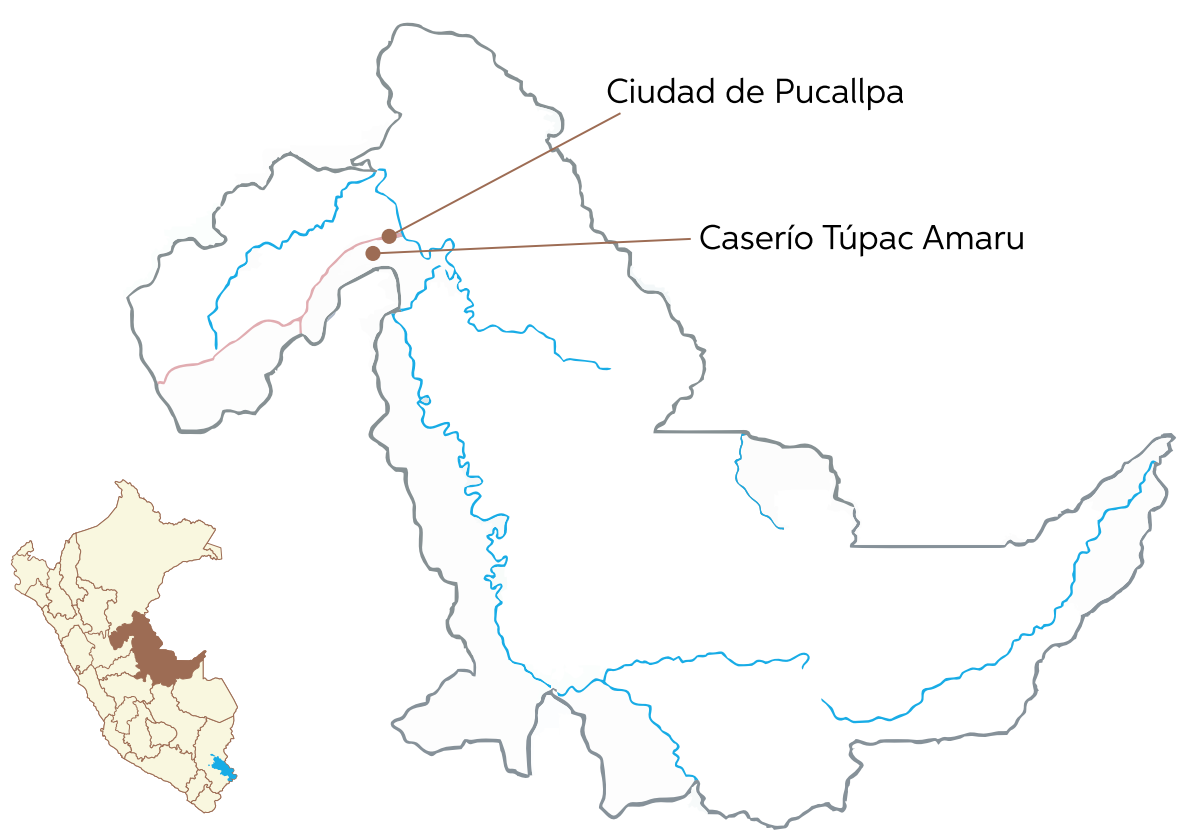

Figura 1: Ubicación del área de estudio y levantamiento de datos en el departamento de Ucayali, Perú. 
Dichas encuestas se elaboraron con un total de 45 preguntas, repartidas en once actividades centrales durante el ciclo de vida de los productos a base de aguaje: 1 . transporte inicial (caserío-aguajal); 2. extracción del fruto; 3. desgranado y envasado; 4. transporte primario (aguajal-caserío); 5. almacenamiento (caserío); 6. transporte secundario (caserío-Pucallpa); 7. almacenamiento y comercializador mayorista; 8 . transporte terciario (comercializador mayoristaminorista); 9. almacenamiento-comercializador minorista; 10. preparación de bebida a base de aguaje y 11. preparación de helado a base de aguaje, con un total de 46 encuestados (Figura 2).
Así mismo, se consignaron datos generales de los encuestados, equipos y maquinarias en las fases productivas, sobre todo el consumo de combustible fósil y de electricidad de cada equipo o maquinaria, insumos para la preparación de los productos, detallando los ingredientes que se usan para la preparación de la bebida y el helado a base de aguaje y otras fuentes de emisión de GEI, abordando temas que se pudieran no haber detallado en los ítems anteriores, tal como la producción de desperdicios producto de la extracción del fruto y la basura producto de la venta de la fruta, la preparación de la bebida y el helado a base de aguaje.

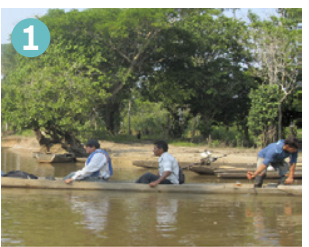

Transporte inicial

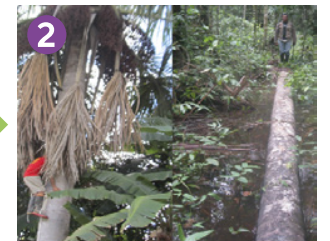

Extracción del aguaje

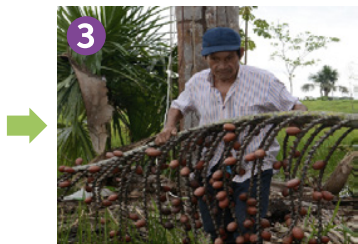

Desgranado de aguaje

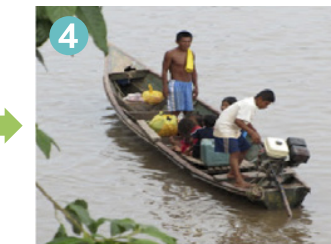

Transporte primario

\section{Fuente de emisiones de GEI}

Combustión fósil

Consumo eléctrico

Residuos sólidos

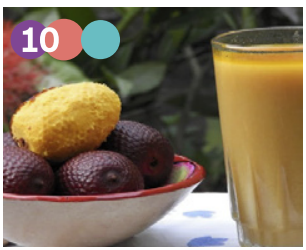

Preparado y venta de helado

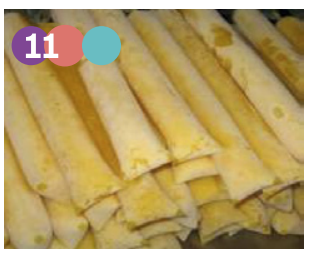

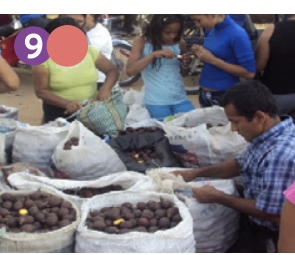

Almacenamiento minorista

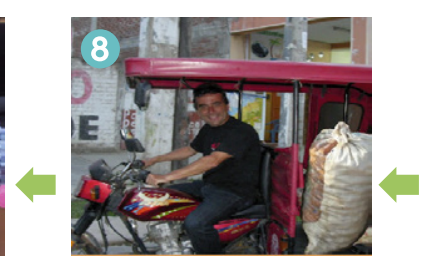

Transporte terciario

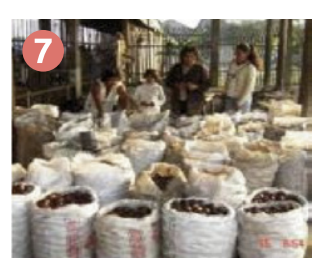

Almacenamiento mayorista

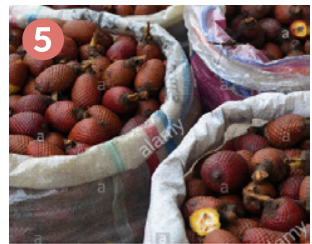

Almacenamiento en el caserío

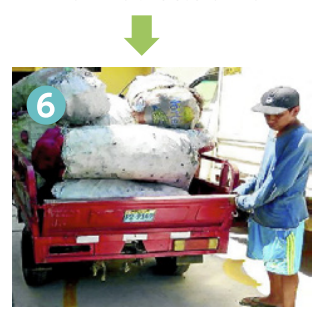

Transporte secundario

Figura 2. Flujo de producción y fuente de emisiones de GEl de la venta del fruto, la producción de bebidas y helados de aguaje (Mauritia flexuosa). 


\section{CUANTIFICACIÓN DE LAS EMISIONES}

Para crear una contabilidad exacta de las emisiones de la producción del fruto, bebida y helado a base de aguaje, se dividió el total de sus emisiones en varias categorías específicas según sus alcances:

Alcance 1. Calculadas en base a las cantidades de la adquisición de combustibles comerciales que se encuentran bajo su control, tales como: 1. combustión fija: producidas por generadores de electricidad, balones de gas, motobomba, etc; 2. combustión móvil: combustión de combustibles en medios de transporte y otros como moto taxis, embarcaciones, motosierras, motoguadañas, etc; 3. emisiones por residuos sólidos: principalmente ocasionados por residuos de basura y desechos orgánicos como hojas secas y ramas (producto de la extracción y otros procesos).

Alcance 2. Se calcularon a partir del consumo de electricidad proveniente de la red pública que suministra electricidad a los lugares de almacenaje, locales comerciales y centros de producción de bebida y helado a base de aguaje.

Alcance 3. Se calcularon a partir de la cantidad de insumos e ingredientes que se usan para la preparación de la bebida y el helado a base de aguaje (agua, azúcar, leche, etc.).
Las emisiones fueron calculadas para las diferentes etapas:

Emisiones por extracción. El cálculo de estas emisiones se inició con la obtención de información referida a todas las actividades durante momento de la extracción del fruto de aguaje, tales como las herramientas utilizadas: hacha y arnés escalador y/u otros que requieren algún tipo de combustible para sufuncionamiento; igualmente se colectó información sobre datos que coadyuvan al cálculo de las emisiones de GEI, como altura de la palmera, cantidad de racimos y frutos por palmera.

Este cálculo requiere especial atención, dado que la extracción se hace de dos formas diferentes: 1 . talando la palmera y 2 . escalando la palmera. Para la estimación de las emisiones de GEI del primer caso se consideró como emisión al GEI que libera la biomasa de las palmeras taladas en las condiciones existentes en un humedal; esta biomasa se calculó usando la fórmula propuesta por Goodman et al. (2013), que requiere la altura total de la palmera de aguaje, seguidamente, se multiplicó esta biomasa por la relación biomasa/ carbono reportada también por Goodman et al. (2013). Posteriormente se multiplicó por el factor de descomposición propuesto por Chimner

Tabla 1: Factores de ajuste y formulas utilizadas para el cálculo de las emisiones de GEl por la tala de la palmera de aguaje (Mauritia flexuosa).

\begin{tabular}{llcccc}
\hline Id & Descripción & $\begin{array}{c}\text { Dato y Unidad } \\
\text { requerida }\end{array}$ & $\begin{array}{c}\text { Ecuación } \\
\text { / Factor de emisión }\end{array}$ & $\begin{array}{c}\text { Unidad } \\
\text { resultante }\end{array}$ & Fuente: \\
\hline 1 & Biomasa de palmeras de aguaje & Altura total $(\mathrm{m})$ & $-261,1+39,068\left(\mathrm{H}_{\text {tot }}\right)$ & $\mathrm{Kg}$ biomasa & $\begin{array}{c}\text { Goodman et al. } \\
(2013)\end{array}$ \\
2 & Relación biomasa / carbono & Biomasa $(\mathrm{Kg})$ & 0,4887 & $\mathrm{Kg} \mathrm{C}$ & $\begin{array}{c}\text { Goodman et al. } \\
(2013)\end{array}$ \\
3 & Carbono descompuesto liberable & Carbono (Kg) & 0,20 & $\mathrm{Kg} \mathrm{C}$ & $\begin{array}{c}\text { Chimner \& Ewel } \\
(2005)\end{array}$ \\
4 & Relación carbono / $\mathrm{CO}_{2}$ & Carbono $(\mathrm{Kg})$ & 3,67 & $\mathrm{Kg} \mathrm{CO}_{2}$ & Rügnitz (2009) \\
\hline
\end{tabular}


Tabla 2: Factores de emisión utilizados para el cálculo de las emisiones de GEl del fruto de aguaje, bebida y helado a base de aguaje.

\begin{tabular}{|c|c|c|c|c|c|}
\hline Id & Descripción & $\begin{array}{l}\text { Dato y unidad } \\
\text { requerida }\end{array}$ & $\begin{array}{c}\text { Ecuación } \\
\text { / Factor de emisión }\end{array}$ & $\begin{array}{l}\text { Unidad } \\
\text { resultante }\end{array}$ & Fuente: \\
\hline 1 & Energía eléctrica (red pública) & Kw.h & 0,5898 & $\mathrm{kgCO}_{2} \mathrm{eq}$ & DEFRA (2012) \\
\hline 2 & Gasolina & $L$ & 2,27 & $\mathrm{kgCO}_{2} \mathrm{eq}$ & IPCC (2006) \\
\hline 3 & $\begin{array}{l}\text { Residuos (preparación de } \\
\text { alimentos) }\end{array}$ & $\mathrm{kg}$ & 0,45 & $\mathrm{kgCO}_{2} \mathrm{eq}$ & DEFRA (2011) \\
\hline 4 & $\begin{array}{l}\text { Residuos de áreas verdes } \\
\text { (hojarasca) }\end{array}$ & $\mathrm{kg}$ & 0,15 & $\mathrm{kgCO}_{2} \mathrm{eq}$ & IPCC (2006) \\
\hline 5 & Azúcar & $\mathrm{kg}$ & 0,307 & $\mathrm{kgCO}_{2} \mathrm{eq}$ & Rein (2010) \\
\hline 6 & Agua & $m^{3}$ & 0,788 & $\mathrm{kgCO}_{2} \mathrm{eq}$ & $\begin{array}{l}\text { Cámara de } \\
\text { agricultura de } \\
\text { Aragón (2019). }\end{array}$ \\
\hline 7 & Leche & L & 0,54 & $\mathrm{kgCO}_{2} \mathrm{eq}$ & $\begin{array}{c}\text { Chávez \& Meléndez } \\
\text { (2016) }\end{array}$ \\
\hline
\end{tabular}

\& Ewel (2005), es decir, del total del carbono cuánto se queda en el ecosistema y cuánto se libera a la atmósfera (20\% se libera). Finalmente se realizó la conversión del carbono total que se emite, que equivale a 3,67 t $\mathrm{CO}_{2}$ por $1 \mathrm{t} \mathrm{C}$ (Rügnitz et al., 2009) (Tabla 1).

Emisiones por transporte. Para el cálculo de estas emisiones se procedió con la obtención de las cantidades de combustible que se usan para el transporte primario, secundarioy terciario dentro de los distritos Callería y Manantay; desde la zona de extracción, almacenado y comercialización, hasta el punto final de venta, teniendo en cuenta el tipo de vehículo que transportó los productos.

Una vez obtenido el dato de consumo se procedió a convertir las unidades a las requeridas por la fórmula de emisión seleccionada, por ejemplo: el consumo de gasolina se obtuvo en galones (gl), sin embargo, la unidad requerida por la fórmula de emisión es litros (L), para lo cual se usó el factor de conversión a galón U.S. de 3,78528 L por gl.

Emisiones por almacenaje. Se definieron las operaciones de almacenaje, tales como las que ocurren en las casas de los productores en el caserío Túpac Amaru, en los almacenes del mayorista y en los almacenes del minorista. Para el cálculo de estas emisiones se cuantificó principalmente el consumo mensual de energía eléctrica en las casas de los productores y los almacenes de los mayoristas y minoristas.

Estos datos también fueron recogidos con la ayuda de encuestas dirigidas a los responsables del almacenaje.

Emisiones por producción de bebida $y$ helado a base de aguaje. Se calcularon a partir de la cantidad de consumo de combustibles fósiles de índole principalmente móvil, residuos de la etapa productiva, tanto orgánicos como inorgánicos, consumo de electricidad e insumos que se usan para la preparación de la bebida (localmente llamada "aguajina"), y el helado a base de aguaje, tales como: agua, azúcar, leche, etc.

Una vez obtenido el dato se procedió a convertir las unidades a las que requiere el factor de emisión seleccionado, por ejemplo: los datos de producción de residuos se obtuvieron en la cantidad de bolsas diarias. Los factores de emisión seleccionados se detallan en la Tabla 2. 


\section{CÁLCULO DE EMISIONES TOTALES}

Una vez realizada la recolección de los datos de campo, se elaboró una base de datos en el programa Excel, uniformizando las unidades de medida a las requeridas por los factores de emisión seleccionados, obteniéndose la cantidad de emisiones de $\mathrm{CO}_{2} \mathrm{eq}$, aplicando la fórmula propuesta por Jiménez et al. (2011).

\section{$\mathrm{Ei}=\mathrm{DA}{ }^{*} \mathrm{FEi}$}

Donde:

$\mathbf{E i}=$ Emisión de la sustancia i expresada en kilogramos de dióxido de carbono equivalente $\left(\mathrm{kgCO}_{2} \mathrm{eq}\right)$.

DA = Dato de actividad, es decir, parámetro que define el grado de actividad de la instalación (unidades de masa, volumen, kilowatt hora (Kw.h), kilometro $(\mathrm{km})$ ) y al que se encuentra referido el factor de emisión correspondiente (Por ejemplo: cantidad de combustible usado, cantidad de electricidad consumida y cantidad de residuos generados).

$\mathbf{F E i}$ = Factor de emisión de la sustancia i, que se define como la cantidad de la sustancia i emitida por cada unidad del parámetro DA.

Una vez realizada la cuantificación de las emisiones, se documentó por separado según emisiones directas e indirectas de GEI para los alcances 1,2 y 3 , según sea el caso.

Para determinar la totalidad de emisiones de GEI por unidad de producto, en el caso de los frutos de aguaje, se procedió a dividir el resultado total obtenido de las emisiones de GEI de los encuestados, entre la cantidad de frutos de aguaje que cada palmera produce en promedio. Para ello se usó la fórmula propuesta por Barrientos \& Molina (2014).

$$
\mathrm{HdC}=\mathrm{ET} / \mathrm{CT}
$$

Dónde:

HdC: Huella de Carbono del producto.

ET: Emisiones totales de GEI, en $\mathrm{kgCO}_{2}$ eq

CT: Cantidad promedio de frutos producidos por productor.

Así mismo, se utilizaron los $\mathrm{kgCO}_{2}$ eq como unidad de medida final para el procesamiento de las emisiones de GEI.

\section{RESULTADOS}

Del total de los encuestados ( $\mathrm{n}=45)$, el $56 \%$ de las personas fueron de sexo femenino y el $44 \%$ de sexo masculino, siendo las fases del 1 al 6 en la producción de aguaje ejecutadas por el sexo masculino (100\%), mientras las fases 8 (73\%) y 9 (93\%) principalmente ejecutadas por el sexo femenino (Figura 2). Es decir, la etapa extractiva y de transporte primario es realizada por el sexo masculino, mientras que las fases de almacenamiento y comercialización son realizadas por el sexo femenino.

\section{EMISIONES DE GEI POR PRODUCCIÓN DE FRUTO DE AGUAJE}

Del total de encuestados $(n=15)$ dedicados a la producción de frutos, el $77 \%$ usa bote motor (peque peque) y el 13\% usa remo. El 77\% tala la palmera usando un hacha para obtener el fruto del aguaje, mientras que el $13 \%$ escala la palmera. Así mismo, los productores de aguaje talan en promedio 55,43 palmeras por cada campaña anual, que dura 3 meses y 6 días en los meses de septiembre a noviembre.

Sobre las emisiones de GEI en el presente estudio se dividió la fase productiva en 3 etapas: 1. Extracción; 2. trasporte y 3. almacenaje, obteniéndose un total de emisiones de: 0,05873 $( \pm 0,008134) \mathrm{KgCO}_{2}$ eq para la extracción talando la palmera, 0,01141 $( \pm 0,002208) \mathrm{KgCO}_{2}$ eq para el transporte y 0,00017 $( \pm 0,000051) \mathrm{KgCO}_{2}$ eq 
para el almacenaje, haciendo un total de 0,07031 $\mathrm{KgCO}_{2}$ eq $\left(\mathrm{R}^{2}=0,66\right.$ y $\left.\mathrm{R}^{2} \mathrm{aj}=0,64\right)$ por cada fruto de aguaje puesto en los centros de venta minoristas de la ciudad de Pucallpa (Figura 3).

Se verificó, según la prueba de Scott \& Knott $(p \leq 0,05)$, la presencia de diferencias estadísticas significativas entre la fuente de emisión de GEI extracción del fruto en comparación al transporte y el almacenaje, entre tanto, estas últimas mostraron resultados similares (Figura 3).

Realizando el cálculo, podemos encontrar diferencias entre las dos formas de extraer el fruto de aguaje, talando la palmera y escalando. Ajustando el cálculo de las emisiones, resalta la diferencia al extraer un fruto sin talar la palmera, el cual reduce su huella de carbono en un 83,53\% quedando en $0,001158 \mathrm{KgCO}_{2}$ eq por fruto de aguaje sin talar la palmera.

\section{EMISIONES DE GEI POR PRODUCCIÓN DE BEBIDA A BASE DE AGUAJE}

Del total de encuestados $(n=15)$ dedicados a la producción y comercialización de la bebida a base de aguaje, se determinó que el 100\% elabora su propia pulpa de aguaje, el $77 \%$ usa su domicilio para el almacenaje y el 13\% no almacena productos (compra lo que vende en el día), el 77 \% compra el hielo y el $13 \%$ lo elabora en su propio domicilio. Finalmente, el 100\% no da ningún tratamiento a sus residuos.

Las emisiones proceden principalmente de los insumos necesarios para la elaboración de la bebida a base de aguaje, para calcularlas se consideró la preparación de un recipiente de 20 L, el cual rinde 80 vasos de $250 \mathrm{ml}$. Del cálculo de las emisiones de GEI se obtuvo que la mayor fuente de emisión derivaba del uso de frutos talando la palmera con 0,71775 $( \pm 0,01697)$ $\mathrm{KgCO}_{2} \mathrm{eq}$, seguido del uso de frutos sin talar la palmera con 0,11820 $( \pm 0,00280) \mathrm{KgCO}_{2}$ eq; en tercer orden, la generación de residuos con 0,03063 (₫0,00572) $\mathrm{KgCO}_{2}$ eq, el uso de azúcar

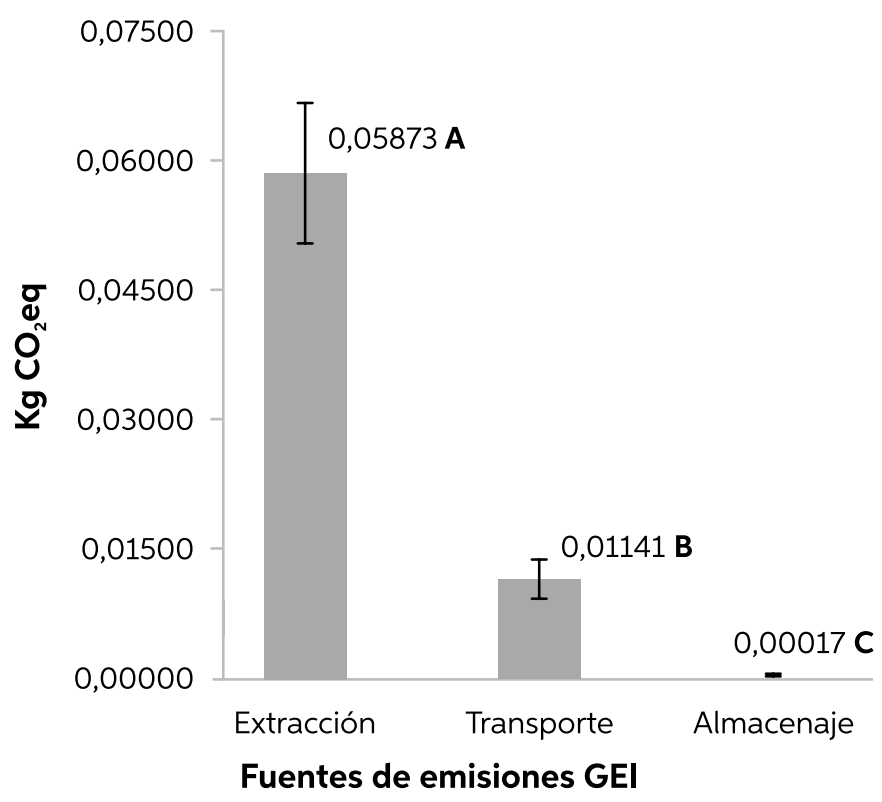

Figura 3: Emisión por fuente en la producción de aguaje (Mauritia flexuosa) proveniente del caserío Túpac Amaru (Departamento de Ucayali, Perú). Las letras iguales en las barras no presentan diferencias estadísticamente significativas según la prueba de Scott \& Knott $(p \leq 0,05)$.

con $0,01535( \pm 0,0000) \mathrm{KgCO}_{2}$ eq, el uso de hielo con 0,00637 ( $\pm 0,00072) \mathrm{KgCO}_{2}$ eq, el uso de congeladora con $0,00477( \pm 0,00184) \mathrm{KgCO}_{2} \mathrm{eq}$, $\mathrm{y}$ finalmente el consumo de agua con 0,00010 $( \pm 0,0000) \mathrm{KgCO}_{2}$ eq. Como es evidente el uso de los frutos de aguaje provenientes de las palmeras taladas para la extracción del fruto muestra el mayor porcentaje de emisiones con un 80,35\%, el resto de fuentes de emisión hacen en su conjunto un total de 19.65\% (Figura 4). La huella de carbono de la producción y comercialización de un vaso de $250 \mathrm{ml}$ de bebida a base de aguaje fue de 0,89317 $\mathrm{KgCO}_{2}$ eq $\left(\mathrm{R}^{2}=0,99\right.$ y $\left.\mathrm{R}^{2} \mathrm{aj}=0,99\right)$.

Se verificó, según la prueba de Scott \& Knott $(p \leq 0,05)$, la presencia de diferencias estadísticas significativas entre las fuentes de emisión de GEI uso de frutos talando la palmera, frutos sin talar la palmera y generación de residuos. Las demás fuentes de emisión mostraron resultados similares (Figura 4). 


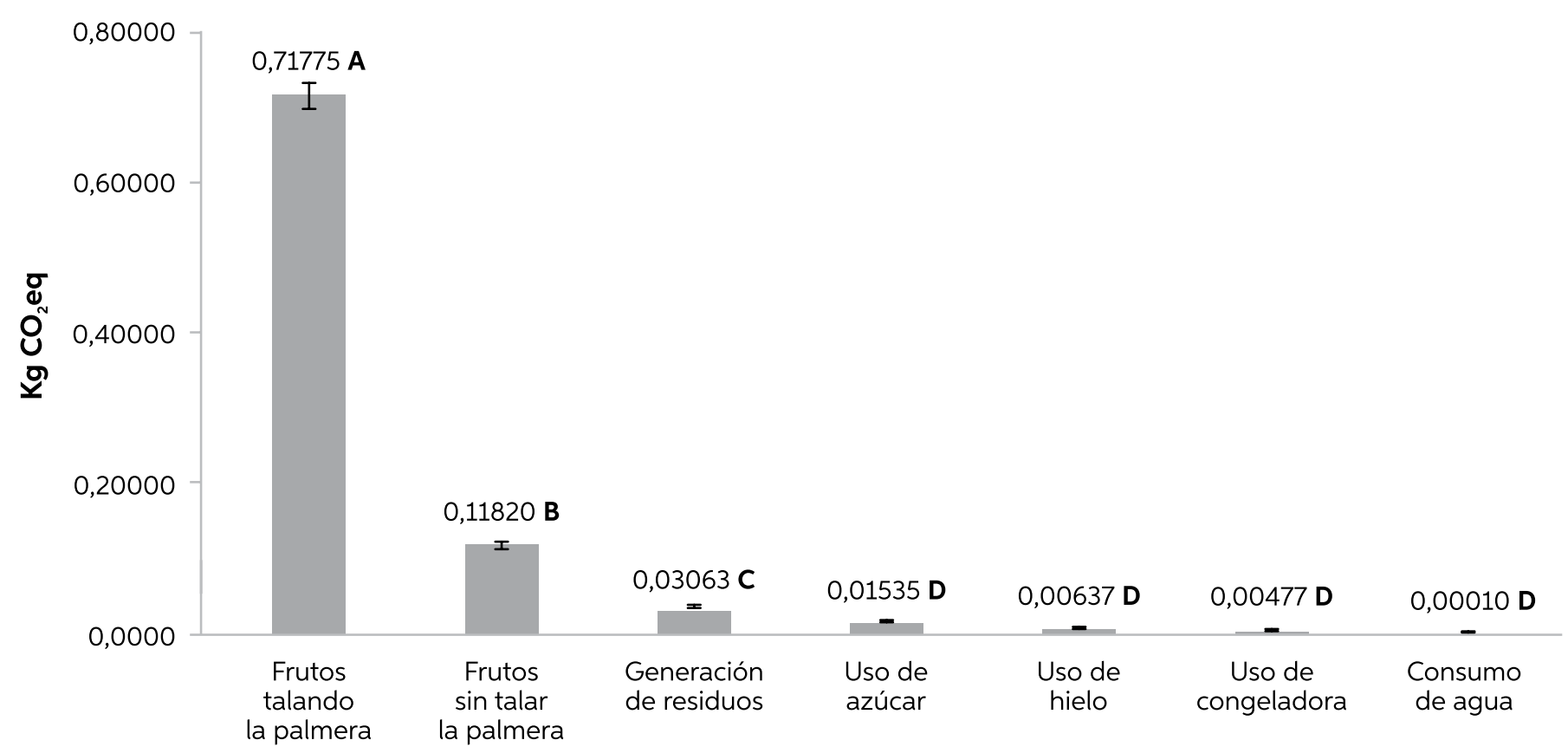

Fuentes de emisiones GEI

Figura 4: Emisiones de GEl para la preparación y comercialización de bebida a base de aguaje (Mauritia flexuosa) en la ciudad de Pucallpa, en las barras las letras iguales no presentan diferencias estadísticamente significativas según la prueba de Scott \& Knott $(p \leq 0,05)$.

\section{EMISIONES DE GEI POR PRODUCCIÓN DE} HELADO A BASE DE AGUAJE

Para la obtención de la huella de carbono de la producción de helado de aguaje se trabajó con una fábrica de helados en la ciudad de Pucallpa, trabajándose como un estudio de caso. Sobre las emisiones producidas, la mayor fuente de emisión fue la de insumos, con una emisión de 0,0842 $\mathrm{KgCO}_{2}$ eq $(92,94 \%)$, seguido de los residuos con una emisión de $0,0034 \mathrm{KgCO}_{2} \mathrm{eq}$ $(3,77 \%)$, en tercer lugar se encuentra la quema de combustibles fósiles con una emisión de 0,0018 $\mathrm{KgCO}_{2}$ eq $(2,04 \%)$ y finalmente el consumo de electricidad con una emisión de $0,0011 \mathrm{KgCO}_{2}$ eq $(1,25 \%)$; detallando las emisiones por insumos (agua, azúcar, leche y frutos de aguaje), se obtiene que la mayor fuente de emisiones deriva del uso de los frutos de aguaje provenientes de las palmeras taladas para la extracción del fruto, con un $95,84 \%$ de esas emisiones.

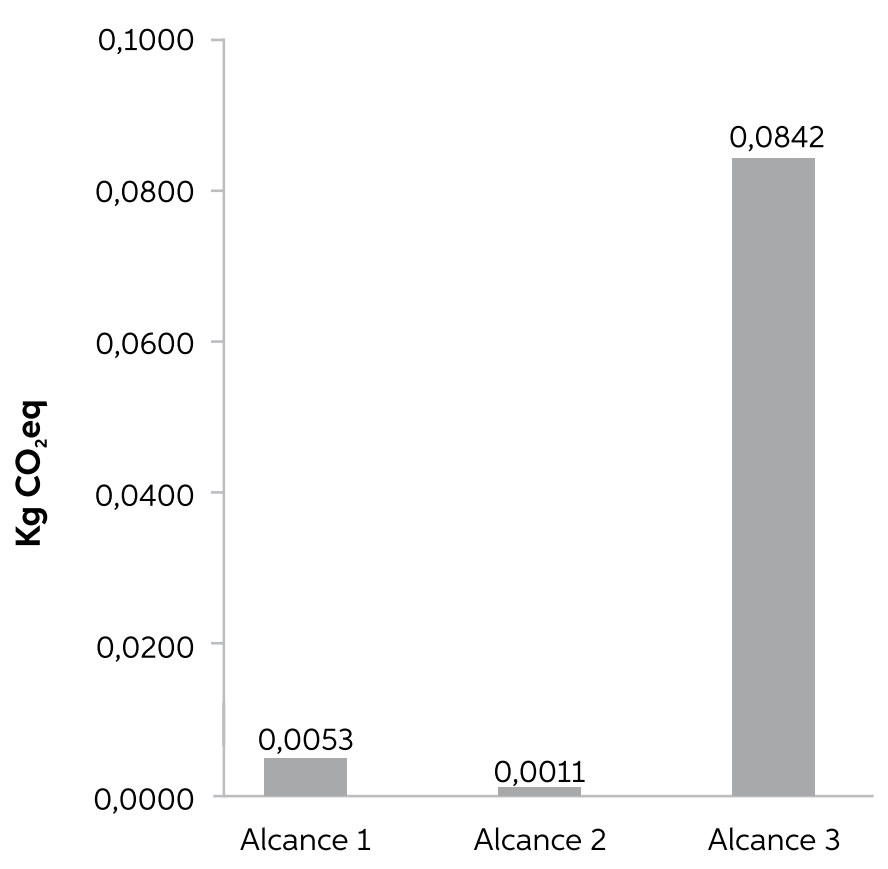

Figura 5. Emisiones de GEl por alcances para la producción de helado a base de aguaje (Mauritia flexuosa) en la ciudad de Pucallpa. 
Agrupando las emisiones por los alcances, se obtiene que: el alcance 1 emite $0,0053 \mathrm{KgCO}_{2}$ eq, el alcance 2 emite $0,0011 \mathrm{KgCO}_{2}$ eq y el alcance 3 emite 0,0823 $\mathrm{KgCO}_{2}$ eq (Figura 5). La huella de carbono de la producción de un helado de $75 \mathrm{ml}$ a base de aguaje fue de 0,0887 $\mathrm{KgCO}_{2}$ eq.

\section{DISCUSIÓN}

La producción de $1 \mathrm{~L}$ de aguajina reportó un total de 3,57 $\mathrm{KgCO}_{2}$ eq, cifra que resulta mayor a la huella de carbono del jugo de naranja que es de 1,7 $\mathrm{KgCO}_{2}$ eq (Environmentalleader.com, 2009), esto se debe principalmente a que en la huella de carbono del jugo de naranja no se consideró el impacto generado por la deforestación en las áreas donde se instala la plantación de este cítrico, además, para extraer la naranja no se tiene que talar el árbol. Sin embargo, si tomamos en cuenta la huella de carbono de $1 \mathrm{~L}$ de aguajina preparada con frutos de aguaje sin talar la palmera, la huella de carbono resultante es $0,7017 \mathrm{KgCO}_{2}$ eq, siendo menor a lo reportado para el jugo de naranja. Comparándolo con la producción de 1 L de leche, la huella de carbono reportada por Chávez \& Meléndez (2016), fue de 0,54 $\mathrm{kgCO}_{2}$ eq, es muy similar a la encontrada en el presente estudio para la producción de aguajina sin talar la palmera.

Un kilogramo de pulpa de aguaje (extraída de 64 frutos de aguaje), presenta una huella de carbono de 4,5215 $\mathrm{KgCO}_{2}$ eq. Si comparamos los resultados reportados por Reijnders \& Huijbregts (2008) en el sudeste asiático sobre la huella de carbono de una planta de extracción de aceite de palma, donde se reporta el rango de 2,8-19,7 kg $\mathrm{CO}_{2}$ eq por kg de aceite crudo de palma, incluyendo el cambio de uso de la tierra, la combustión de combustibles fósiles y el tratamiento de aguas residuales en su análisis, resultan mayores a los encontrados en este estudio. Si comparamos lo reportado por Poroma (2012) sobre la producción y comercialización de 1 kg de cacao, donde se genera una emisión de 4,98 $\mathrm{KgCO}_{2}$ eq a la atmosfera, los resultados son muy similares a los encontrados en el presente estudio.

Sin embargo, la huella de carbono de $1 \mathrm{Kg}$ de pulpa de aguaje fue inferior a la del café, que reporta un $8,4 \mathrm{kgCO}_{2} \mathrm{eq}$ (PCF, 2008) y superior a la huella de la producción de café orgánico con $0,1 \mathrm{KgCO}_{2}$ eq y a la del café convencional con $0,33 \mathrm{KgCO}_{2}$ eq de la cooperativa Triunfo Verde en México (Aguilar, 2012).

Actualmente existe una falta de estudios sobre la evaluación del ciclo de vida de helados a nivel mundial, los estudios existentes se centran en el potencial de calentamiento global Konstantas et al. (2018).

Dicho esto, en el presente estudio se reporta que la producción de $1 \mathrm{Kg}$ de helado de aguaje tiene una huella de carbono de 1,2083 $\mathrm{KgCO}_{2} \mathrm{eq}$, lo que se encuentra por debajo de lo reportado por Ben \& Jerry's (2016), con un rango de 3,66$3,94 \mathrm{kgCO}_{2}$ eq por $1 \mathrm{Kg}$, al igual que lo reportado por Scottish Government (2011) con 4,0 $\mathrm{kgCO}_{2} \mathrm{eq}$ por $1 \mathrm{Kg}$. El único reporte que presenta valores inferiores es el de Foster et al. (2006), con 0,97 $\mathrm{KgCO}_{2}$ eq por $1 \mathrm{Kg}$. Estos valores difieren por los insumos que cada helado necesita para su preparación, en este estudio el fruto de aguaje es la base, en otros es el cacao. Además, no se incluye el manejo de residuos por varios años y la venta minorista (Konstantas et al., 2018).

De igual manera, en cuanto a la diferencia de la huella de carbono entre el helado a base de aguaje $\mathrm{y}$ el vaso de aguajina, que reportan 0,89317 y 0,0906 $\mathrm{KgCO}_{2} \mathrm{eq}$, respectivamente, esta se debe principalmente a los ingredientes con los cuales se prepara la aguajina, requiriéndose alrededor de 100\% más de frutos de aguaje que en la preparación del helado, además, el vaso tiene mayor cantidad de volumen, $250 \mathrm{ml}$ comparado con los $75 \mathrm{ml}$ de cada helado. 


\section{REFERENCIAS BIBLIOGRÁFICAS}

Aguilar, J. 2012. Impacto socioeconómico y ambiental de la certificación orgánica-comercio justo de café (Coffea arabica) en la Región Frailesca, Chiapas, México. Tesis de post-grado. Centro Agronómico Tropical de Investigación y Enseñanza (CATIE), Costa Rica, Turrialba, 175pp.

Barrientos, E.; Molina, M. 2014. Medida dela huella de carbono en una empresa de fabricación de briquetas. Tesis de pre-grado. Universidad Nacional Agraria la Molina (UNALM), Lima, Perú, 116pp.

Ben \& Jerry's. 2016. The footprint of your pint. (https://www.benjerry.com/values/issueswe-care-about/climate-justice/life-cycleanalysis). Acceso: 11/06/2019.

Cámara de agricultura de Aragón. 2019. Cálculo automático de emisiones totales en relación a los consumos energéticos de sus instalaciones. (https://www.camarazaragoza.com/wpcontent/uploads/2012/10/calculoemisiones. xls). Acceso: 09/08/2019.

Chávez, J.; Meléndez, P. 2016. Huella de carbono parcial de un kilo de leche pasteurizada de la planta piloto de leche de la UNALM. Tesis de pre-grado, Universidad Nacional Agraria la Molina (UNALM), Lima, Perú. 93pp.

Chimner, R.; Ewel, K. 2005. A tropical freshwater wetland: II. Production, decomposition, and peat formation. Wetlands Ecology and Management. 13: 671-684. DOI: https://doi. org/10.1007/s11273-005-0965-9

Del Castillo, D.; Otárola, E.; Freitas, L. 2006. Aguaje, la maravillosa palmera de la Amazonía. Instituto de Investigaciones de la Amazonía Peruana. Iquitos. $51 \mathrm{pp}$.

Delgado, C.; Couturier G.; Mejía, K. 2007. Mauritia flexuosa (Arecaceae: Calamoideae), an amazonian palm with cultivation purposes in
Peru. Fruits, 62: 157-159. DOI: https://doi. org/10.1051/fruits:2007011

DEFRA (Department for Environment, Food and Rural Affairs). 2008. Guidelines to Defra's GHG conversion factors: Methodology paper for transport emission factors, Departament for Environment, Food and Rural Affairs. (http://www.co2nnect.org/help_sheets/?op_ id=602\&opt_id=98\&nmlpreflang=es). Acceso: 14/06/2019.

DEFRA (Department for Environment, Food and Rural Affairs). 2011. Guidelines to Defra / DECC's GHG conversion factors for company reporting: Methodology paper for emission factors. (https://www.gov.uk/government/ uploads/system/uploads/attachment_data/ file /69314/pb13625-emission-factormethodology-paper-110905.pdf). Acceso: 18/04/2019.

DEFRA (Department for Environment, Food and Rural Affairs). 2012. Guidelines to Defra / DECC's GHG conversion factors for company reporting: Methodology paper for emission factors. (https://www.gov.uk/government/ uploads/system/uploads/attachment_data/ file/69568/pb13792-emission-factormethodology-paper-120706.pdf). Acceso: 09/08/2019.

Environmentalleader.com. 2009. Carbon footprint of tropicana orange juice. Environmental + energy leader. (https://www. environmentalleader.com/2009/01/carbonfootprint-of-tropicana-orange-juice-17-kg/). Acceso: 09/09/2019.

Foster, C.; Green, K.; Bleda, M.; Dewick, P.; Evans, B.; Flynn, A.; Mylan, J. 2006. Environmental impacts of food production and consumption: A report to the Department for Environment, Food and Rural Affairs. Manchester Business School, University of Manchester, London. 199pp. 
Freitas L.; Pinedo, M.; Linares, C. 2006. Descriptores para el aguaje (Mauritia flexuosa L. f.), Instituto de Investigaciones de la Amazonía Peruana. Iquitos. 33pp.

García, D.; Honorio, E.; Del Castillo, D. 2012. Determinación del stock de carbono en aguajales de la cuenca del río Aguaytía, Ucayali -Perú. Folia Amazónica, 21(1-2), 153-160. DOI: https://doi.org/10.24841/fa.v21i1-2.43

Goodman, C.; Phillips. O.; Del Castillo, D.; Freitas, L.; Tapia, S.; Monteagudo, A.; Baker, T. 2013. Amazon palm biomass and allometry. Forest Ecology and Management, 310: 994-1004. DOI: https://doi.org/10.1016/j. foreco.2013.09.045

IPCC. 2006. IPCC guidelines for national greenhouse gas Inventories, prepared by the National Greenhouse Gas Inventories Programme. Institute for Global Environmental Strategies (IGES), Tokyo. 18pp.

Jiménez, L.; De La Cruz, J.; Carballo, A.; Domench, J. 2011. Enfoques metodológicos para el cálculo de la huella de carbono, Observatorio de la Sostenibilidad en España (OSE). (http://www.carbonfeel.org/Carbonfeel_2/ Bitacora/Entradas/2011/9/15_Informe_ Enfoques_metodologicos_para_el_caclulo_ de_la_Huella_de_Carbono_del_Isntituo_de_la_ Sostenibilidad_en_Espana_files/Informe $\% 20$ OSE.pdf). Acceso: 17/04/2019.

Konstantas, A.; Stamford, L.; Azapagic, A. 2018. Environmental impacts of ice cream. Journal of CleanerProduction, 209:259-272.DOI: https:// doi.org/10.1016/j.jclepro.2018.10.237

Lash, J.; Wellington, F. 2007. Competitive advantage on a warming planet. Harvard Business Review, 85(3): 94-102.

Leontief, W. 1986. Input-output economics, 2n ed., Oxford University Press, New York. 436pp. Licona, A.; Ramírez, E. 2014. China y Estados Unidos, compromisos para mitigar las emisiones de gases de efecto invernadero. Revista Cimexus, 9(1): 91-104.

Matthews, H.; Hendrickson, C.; Weber, C. 2008. The importance of carbon footprint estimation boundaries. Environmental Science \& Technology, 42(18): 5839-5842. DOI: https:// doi.org/10.1021/es703112w

Ministerio del Ambiente (MINAM).2010. Mapa del patrimonio forestal nacional. (https://sinia. minam.gob.pe/mapas/mapa-patrimonioforestal-nacional). Acceso: 10/12/2019.

OMM (Organización Mundial de Meteorología). 2019. Boletín sobre los gases de efecto invernadero $\mathrm{N}^{\circ} 15$. Estado de los gases de efecto invernadero en la atmósfera según las observaciones mundiales realizadas en 2018. OMM, Ginebra. 8pp.

PCF (Pilot Projekt Deutschland). 2008. Case study Tchibo Private Kaffee Rarity Machare By Tchibo GMBH, PCF Pilot Project, Berlin. 38pp.

Poroma, D. 2012. Estrategia de reducción de la huella de carbono en la producción del grano de cacao (Theobroma cacao L.) para la cooperativa CACAONICA en Waslala, Nicaragua. Tesis de post-grado. Centro Agronómico Tropical de Investigación y Enseñanza, Turrialba, Costa Rica. 79pp.

Reijnders, L.; Huijbregts, M. 2008. Palm oil and the emission of carbon-based greenhouse gases. Journal of Cleaner Production, 16: 477-482. DOI: https://doi.org/10.1016/j. jclepro.2006.07.054

Rein, P. W. 2010. The carbon footprint of sugar. Sugar Industry. Zuckerind, 135(7): 427-434. DOI: http://doi.org/10.36961/si10006

Rojas, R.; Ruiz, G.; Ramírez, P. 2001. Comercialización de masa y «fruto verde» de Aguaje (Mauritia Flexuosa L.f.) en Iquitos (Perú). Folia Amazónica, 12(1-2):15-38. DOI: https://doi.org/10.24841/fa.v12i1-2.123

Rügnitz, M. T.; Chacón, M. L.; Porro, R. 2009. Guía para la determinación de carbono en 
pequeñas propiedades rurales. Centro Mundial Agroflorestal (ICRAF) / Consorcio Iniciativa Amazónica (IA), Lima, Perú. 79pp.

Santos, L. 2005. Nutritional and ecological aspects of buriti or aguaje (Mauritia flexuosa Linnaeus filius): a carotene-rich palm fruit from Latin America. Ecology of Food and Nutrition, 44: 1-14. DOI: https://doi. org/10.1080/03670240500253369.

Scottish Government. 2011. Scottish dairy supply chain greenhouse gas emissions. Scottish Government, Edinburgh. 53pp.

Recibido: 11 de setiembre de 2020 Aceptado para publicación: 22 de octubre de 2020 